\title{
A propos de quelques aspects actuels de l'étude des transferts des polluants radioactifs jusqu'aux sites critiques des organismes
}

\author{
R. BITTEL $(*)$, R. MAGNAVAL $(* *)$
}

(Manuscrit reçu le 5 mai 1977)

\begin{abstract}
RÉSUMÉ
Le but de ce travail est de mettre en relief, parmi les divers aspects relatifs aux transferts des radionucléides des points de rejets jusqu'aux sites critiques de l'homme et des organismes du milieu, ceux qui gardent un intérêt actuel, compte tenu de l'évolution prévisible de l'énergie nucléaire. On a mis l'accent, en particulier, sur les transferts à partir des produits bruts récoltés, sur la microlocalisation des radionucléides incorporés et sur les conséquences possibles de la valorisation des déchets pour l'alimentation.
\end{abstract}

\begin{abstract}
Among the various aspects of radionuclide transfers from release points till the critical sites in human and other living organisms this work shows out the transfers of present concern in the light of the predictable evolution of nuclear energy. Transfers from agricultural products, microlocalization of incorporated radionuclides and the possible consequences of the utilization of wastes as food are especially considered.
\end{abstract}

(*) Commissariat à l’Énergie Atomique, Institut de Protection et de Sûreté Nucléaire, Département de Protection, B.P. $n^{\circ}$ 6, 92260 Fontenay-aux-Roses.

(**) Centre National de la Recherche Scientifique, Université de Paris-Sud, Équipe de Nutrition et de Cinétique Cellulaire, rue J.-B.-Clément, 92290 Châtenay-Malabry. 


\section{INTRODUCTION}

Les transferts des polluants radioactifs depuis leur point d'apparition dans le milieu jusqu'aux organes critiques de l'homme ont fait l'objet de très nombreuses études, en raison de l'urgence des problèmes posés par le respect de la santé de l'individu et des groupes de populations et qui pouvaient être des facteurs limitants de l'utilisation industrielle de l'énergie nucléaire. Un ensemble très considérable de travaux a conduit à une quantification des conséquences pour l'homme des rejets des diverses implantations nucléaires. L'accent a, en particulier, été mis :

- sur la notion de facteur de transfert $F$ qui semble constituer a priori un moyen commode de mesurer l'intensité des mouvements des éléments entre deux milieux physiques ou biologiques 1 et 2 , consécutifs ou non

$$
F=\frac{\text { activité spécifique du milieu } 2}{\text { activité spécifique du milieu } 1} ;
$$

- sur le parallélisme existant entre les comportements de divers isotopes stables ou instables d'un même élément;

- sur les cas où ce parallélisme est en défaut et sur l'interprétation de ces cas particuliers, ce qui a conduit à insister sur l'incidence de paramètres physicochimiques, en particulier sur celle de la nature physique ou physicochimique des combinaisons des isotopes en cause.

Il apparaît maintenant souhaitable de faire le point d'un certain nombre d'aspects encore controversés ou qui prennent une importance de plus en plus grande en fonction de l'évolution des techniques nucléaires, du nombre croissant des installations nucléaires et des incidences socio-économiques qui peuvent en résulter $[19,20,26]$.

\section{LES DIVERSES ÉTAPES DES TRANSFERTS}

Classiquement, on envisage les phénomènes de transferts des points de rejets aux organes critiques de l'homme. Mais il apparaît maintenant qu'il faille aller plus loin si l'on veut obtenir des bases solides pour l'optimisation de la radioprotection, en particulier dans le domaine des très faibles doses : il semble souhaitable d'étudier la microlocalisation des radionucléides aux échelles cellulaire, subcellulaire, voire moléculaire. Les diverses étapes des transferts sont donc les suivantes :

- dispersion dans l'un des milieux de l'environnement physique (atmosphère, stratosphère, ionosphère, eaux, sols, sous-sols, formations géologiques);

- transfert de matière entre deux ou plusieurs de ces milieux, par exemple : transfert eaux $\rightarrow$ sols, eaux $\rightarrow$ atmosphère, atmosphère $\rightarrow$ sols et eaux;

- passage de l'environnement physique au milieu biologique et transfert dans les différents échelons des chaînes écologiques; 
- transfert des produits bruts servant de matières premières à l'alimentation de l'homme et des animaux aux aliments au stade de leur consommation;

- entrée des radionucléides chez l'homme et les animaux de ferme;

- localisation des radionucléides dans les organes de l'homme et des animaux de ferme;

- microlocalisation aux échelles cellulaire, subcellulaire et moléculaire dans les organismes.

\section{3. ÉVALUATION DE LA DOSE \\ DÉLIVRÉE A L'ORGANE CRITIQUE DE L'HOMME (contamination après ingestion d'aliments contaminés)}

Deux méthodes ont été proposées pour estimer la dose délivrée du fait de l'ingestion d'aliments contaminés par des radionucléides provenant d'un seul milieu physique (eaux, sols, atmosphère) :

- la méthode dite des facteurs de transfert ou des facteurs de concentration, basée sur la détermination expérimentale in vitro et in situ des facteurs de transfert ou de concentration $[10,11]$;

- la méthode dite de l'activité spécifique, basée sur la constance, à l'équilibre, de l'activité spécifique d'un élément, tout au long de son transfert du milieu aux organes de référence de l'homme $[8,11,30]$.

TABLEAU I

\begin{tabular}{|c|c|c|c|}
\hline \multicolumn{4}{|c|}{$\begin{array}{l}\text { DÉTERMINATION DES NIVEAUX MAXIMAUX ADMISSIBLES POUR UN SITE MARIN } \\
\text { (Sud-Bretagne et Nord-Gascogne, France) [11] }\end{array}$} \\
\hline Radionucléides & $\begin{array}{l}\text { Organes critiques } \\
\text { (homme) }\end{array}$ & $\begin{array}{l}\text { Méthode : facteur } \\
\text { de transfert } \\
\text { (en p(i.g ') }\end{array}$ & $\begin{array}{c}\text { Méthode : activité } \\
\text { spécifique } \\
\left(l \text { en pCi.g }{ }^{-1}\right)\end{array}$ \\
\hline 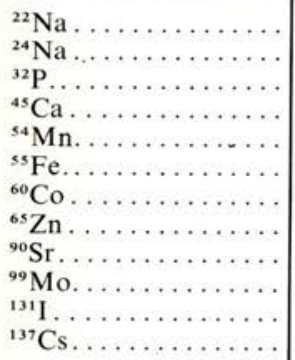 & $\begin{array}{c}\text { Organisme entier } \\
\text { Organisme entier } \\
\text { Squelette } \\
\text { Squelette } \\
\text { Foie } \\
\text { Rate } \\
\text { Organisme entier } \\
\text { Organisme entier } \\
\text { Squelette } \\
\text { Reins } \\
\text { Thyroìde } \\
\text { Organisme entier }\end{array}$ & $\begin{array}{c}1000 \\
10000 \\
0,05 \\
40 \\
0,5 \\
4 \\
3 \\
2 \\
70 \\
100 \\
1 \\
30\end{array}$ & $\begin{array}{c}6000 \\
7000 \\
0,03 \\
2000 \\
6 \\
3 \\
8 \\
2 \\
600 \\
100 \\
10 \\
300\end{array}$ \\
\hline
\end{tabular}

Les principes de ces deux méthodes sont bien connus et ont fait l'objet de discussions nombreuses, échelonnées de 1960 à 1970. On n’y reviendra donc pas

vOL. $12-\mathrm{N}^{\circ} 4$ 
ici, si ce n'est pour insister sur le fait que ces méthodes, en apparence indépendantes, font, en fait, appel aux mêmes hypothèses de base, en particulier au parallélisme entre les comportements des divers isotopes d'un même élément. Le tableau I montre d'ailleurs que, à de rares exceptions près, la détermination des niveaux maximaux admissibles pour l'eau de mer, considérée comme source d'aliments pour l'homme, conduit à des valeurs qui ne diffèrent pas de plus d'un facteur 10 , ce qui paraît une bonne concordance, compte tenu de la difficulté et de l'imprécision des mesures.

\section{REMISE EN SUSPENSION D'ÉLÉMENTS DÉPOSÉS SUR LE SOL ET MÉCANISMES INVERSES}

Il paraît probable que la remise en suspension locale et momentanée, dans la basse atmosphère, de radionucléides récemment déposés sur le sol, est un mécanisme important de pollution des organismes vivants, qu'il s'agisse de l'inhalation des poussières par les animaux et l'homme ou de la rétention, par les parties aériennes des végétaux, des poussières redéposées $[2,3]$. Ceci conduit à étudier avec soin les phénomènes qui s'opposent à cette remise en suspension, c'est-à-dire les phénomènes d'incorporation et de migration dans le sol. Ces deux aspects opposés sont évidemment particulièrement importants lorsqu'il s'agit de radioisotopes réputés peu solubles ou insolubles, en particulier les césiums, les zirconiums, les plutoniums, les transplutoniens et les radioéléments naturels émis à l'état particulaire (mines).

Les mécanismes s'opposant à la remise en suspension sont de divers ordres : ce peut être le résultat d'un compactage de l'horizon superficiel (" roulage » des sols agricoles réduisant fortement la porosité), le résultat de la couverture du sol par du plastique ou même par de la paille, le résultat du prélèvement des radionucléides par les cultures, enfin, et probablement surtout, de l'effet rhizosphérique qui sera étudié plus en détail ici.

La présence de racines vivantes dans le sol implique évidemment l'existence d'un rejet de gaz carbonique (respiration racinaire) qui, dissous dans la solution du sol, y maintient une concentration relativement élevée en ions bicarboniques ( 1 à 20 meq $\mathrm{CO}_{3} \mathrm{H}^{-}$parl) et tend donc à l'acidifier. On trouve, d'autre part, au voisinage immédiat des racines, des concentrations relativement élevées de divers acides organiques qui sont soit exsudés par les racines, soit produits par une microflore particulièrement abondante et active. Il en résulte que le $\mathrm{pH}$ des gangues de sol entourant les racines est parfois significativement inférieur au pH moyen du sol environnant [9]. Il ne faut pas sous-estimer, d'autre part, l'incidence de l'échange direct, sans passage intermédiaire dans la solution du sol, entre les cations adsorbés ou temporairement fixés par le sol et des cations en surface des racines absorbantes $\left(\mathrm{H}^{+}\right.$en particulier). L'ensemble de ces mécanismes favorise l'évolution des formes physicochimiques des radioisotopes 
" insolubles " : pour les moins insolubles et ceux qui sont dans un état physique très divisé, il peut s'agir d'une solubilisation ou tout au moins d'une dépolymérisation relative, mais les mécanismes de complexation et ceux d'incorporation à l'état de molécules organiques semblent avoir une plus grande incidence $[4,6,27,39,40]$. Il convient de remarquer que ces actions n'impliquent pas nécessairement une augmentation des prélèvements racinaires des radioisotopes les plus insolubles, mais qu'elles favorisent l'extension de la pollution vers les couches profondes du sol et les nappes phréatiques par migration verticale, alors qu'elles s'opposent aux migrations horizontales qui sont le fait des phénomènes de resuspension.

\section{INCIDENCE DE PARAMÈTRES PHYSIQUES ET PHYSICOCHIMIQUES}

On se limitera à quelques exemples, car les travaux consacrés à ce problème sont très nombreux. En ce qui concerne la forme physique, on peut mettre l'accent sur le comportement des radioruthéniums en milieu marin [1, 23, 28]. Ainsi, l'algue Corallina officinalis fixe, toutes choses égales par ailleurs, 5 à 10 fois plus de ruthénium sous des formes " peu solubles " que de ruthénium sous forme " soluble » (tableau II). Bien entendu, la « solubilité " des composés du ruthénium dépend de leur état de polymérisation qui, lui-même, est fonction des traitements des effluents avant rejet et, pour une part difficile à apprécier, des

TABLEAU II

\begin{tabular}{|c|c|c|}
\hline \multicolumn{3}{|c|}{$\begin{array}{l}\text { FACTEURS DE TRANSFERT EAU } \rightarrow \text { ORGANISMES MARINS } \\
\text { POUR DIFFÉRENTES FORMES DU RUTHÉNIUM [23] }\end{array}$} \\
\hline & Formes " solubles" & Formes " insolubles" \\
\hline 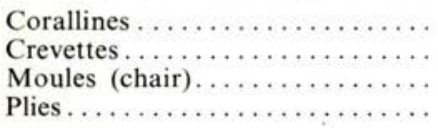 & $\begin{array}{l}89 \\
40 \\
3,9 \\
0,04\end{array}$ & $\begin{array}{l}439 \\
166 \\
35 \\
0,16\end{array}$ \\
\hline
\end{tabular}

paramètres physicochimiques du milieu océanique. Des résultats allant dans le même sens ont été obtenus sur la moule (Mytilus edulis) et la plie (Pleuronectes platessa), non seulement avec les radioruthéniums mais aussi avec le fer 59. Il convient de signaler, cependant, les différences entre le comportement des ruthéniums et celui du fer, après leur rétention par l'organismes :

${ }^{59} \mathrm{Fe}$ est un isotope d'un élément régulé métaboliquement : les formes « insolubles » du fer se répartissent dans tout l'organisme. ${ }^{106} \mathrm{Ru}$ est un isotope d'un élément qui n'est pas régulé (peut-être l'est-il partiellement et indirectement 
par le fer) : ses formes solubles migrent lentement vers les organes internes, alors que les formes insolubles restent immobiles sur les organes directement contaminés (T.G.I., branchies).

L'état physique du plutonium qui peut être libéré dans l'atmosphère à la suite d'accidents à des réacteurs rapides refroidis au sodium semble être différent de celui du plutonium émis à la suite d'autres accidents. Ce fait, d'abord imputé à : un état valenciel particulier du plutonium associé à l'oxyde de sodium, semble plutôt lié à un état de division beaucoup plus grande [32-34].

\section{TABLEAU III}

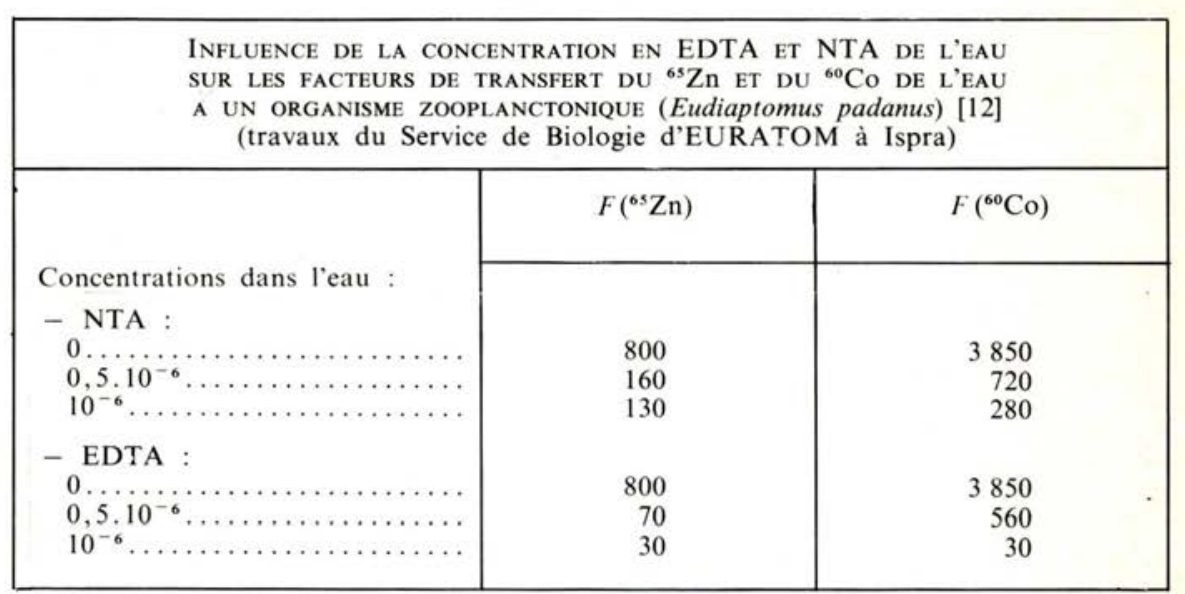

En ce qui concerne l'incidence de l'état physicochimique des radionucléides sur leurs facteurs de transfert, qui a fait l'objet de très nombreux travaux, les recherches poursuivies à Ispra sur les transferts de l'eau à une espèce zooplanctonique dulçaquicole peuvent servir d'illustration. On constate (tableau III) que le cobalt et le zinc complexés soit par l'acide nitrilotriacétique (NTA), soit par l'acide éthylène-diamine-tétracétique (EDTA) passent dans le zooplancton avec des intensités bien moindres que le cobalt et le zinc à l'état ionique simple. Ces recherches ont un débouché direct sur des problèmes pratiques, puisque l'espèce utilisée, Eudiaptomus padanus, entre dans les chaînes trophiques lacustres du nord de l'Italie et que les complexants utilisés sont proposés pour remplacer, dans les détergents commerciaux, les polymétaphosphates jugés responsables de l'eutrophisation des eaux continentales [12].

Parmi les paramètres physicochimiques susceptibles de modifier le transfert aux organismes des radionucléides du milieu, il faut citer la concentration en isotopes stables du milieu (dilution isotopique) et sa concentration en éléments chimiquement et biochimiquement voisins. Le cas des couples $\mathrm{Ca}-\mathrm{Sr}$ et Cs-K est 
bien connu. L'étude de l'incidence de faibles teneurs de cadmium dans l'eau sur le facteur de transfert du zinc 65 (deux éléments chimiquement proches) a mis 'parfois en évidence de tels antagonismes ou tout au moins montré la complexité d'un tel problème $[36,37]$.

Les problèmes soulevés par les rejets thermiques des installations nucléaires, qui ne sont d'ailleurs pas l'apanage de ces seules installations, sont $a$ priori très complexes, ces rejets pouvant à la fois modifier l'équilibre thermique des milieux de rejet, en particulier leur stratification, le métabolisme général des organismes aquatiques, la durée de la période annuelle pendant laquelle l'animal aquatique se nourrit, les équilibres écologiques entre espèces, la forme physicochimique des éléments et des radionucléides et, localement au voisinage des sites des rejets thermiques, la remise en suspension dans l'eau de radionucléides et d'isotopes stables déjà déposés. On se limitera ici encore à quelques exemples pour marquer l'incidence d'une élévation locale de température en milieu océanique :

- chez les crevettes, une élévation locale de la température de l'eau de 10 à $15^{\circ} \mathrm{C}$ double le rythme des mues et multiplie par 1,5 l'absorption de nourriture [42]. Si les radionucléides sont apportés par la nourriture, la contamination de l'animal est multipliée par 1,5; par contre, si l'apport se fait directement à partir de l'eau de mer, l'incidence de variations de la température est nulle ou même négative; seul, subsiste l'effet favorable de l'élévation de température sur la croissance des individus:

- la décontamination naturelle de moules et de blennies contaminées préalablement paraît être d'autant plus intense en présence d'eau de mer non contaminée que la température du milieu est plus élevée [24];

- pour les organismes tropicaux marins filtrant l'eau de mer, il semble qu'il existe une compensation entre l'accélération du rythme de la filtration et l'augmentation de l'élimination biologique de l'élément; dans ces conditions, les effets de variation de température sont relativement faibles et difficiles à interpréter [38]. Des observations allant dans le même sens ont été faites sur des organismes marins européens (moules, blennies) [24];

- l'incidence de rejets thermiques sur la turbulence ou sur la stratification du milieu se pose à la fois en milieu océanique [44] et en milieu atmosphérique $[25,29]$. Ainsi, le rejet d'effluents radioactifs gazeux secs et de vapeur d'eau en des sites voisins peut modifier la turbulence de la basse atmosphère et accroître le lessivage vers le sol de particules radioactives sorbées par le brouillard humide. Il s'agit, là encore, de deux phénomènes dont les effets sont opposés et dont il importe de faire le bilan en fonction des conditions locales.

\section{TRANSFERTS DU PRODUIT BRUT RÉCOLTÉ AU PRODUIT CONSOMMÉ}

Plusieurs étapes sont à considérer :

- la commercialisation et la distribution des produits bruts;

- les technologies agro-alimentaires entre les produits bruts récoltés et les produits consommés;

VOL. $12-\mathrm{N}^{\circ} 4$ 
- la commercialisation et la distribution des produits au stade de leur consommation par l'homme;

- la réutilisation, du fait de l'homme, des " refus » des industries agroalimentaires, en particulier de ceux qui peuvent et pourront ultérieurement être valorisés par différentes actions technologiques et biologiques, notamment par des actions microbiennes (valorisation des déchets).

\section{1. Circuits de COMmerCialisation Et DE Distribution (matières premières des aliments et aliments)}

L'autoconsommation tend à devenir de plus en plus rare. Les produits en provenance d'une région donnée sont maintenant exportés, soit à l'intérieur des frontières d'un pays, soit à l'intérieur de vastes régions internationales. C'est, par exemple, le cas du périple de l'anguille, pêchée en Camargue, fumée aux PaysBas et consommée partout dans la Communauté Européenne. Le lait local subit une dilution importante, au niveau des usines, par des laits d'autres provenances, ce qui a pour résultat de niveler au stade de la distribution et de la consommation les différences qu'on aurait pu observer entre laits de divers producteurs $[18,35]$.

\subsection{TRANSFERTS DES PRODUITS BRUTS AUX PRODUITS CONSOMMÉS}

Il est clair que l'homme ne consomme qu'une fraction des produits bruts récoltés : par exemple, il élimine les feuilles externes des salades, il pèle les fruits... D'autre part, il fait subir aux produits un certain nombre de transformations qui peuvent en modifier la composition. L'incidence de ces pratiques sur les transferts de radionucléides est complexe. On se limitera ici encore à quelques exemples :

\section{Traitement des eaux destinées à la boisson}

Les traitements subis par les eaux brutes en vue d'en faire des eaux utilisables pour la consommation par l'homme sont multiples, mais presque tous les procédés modernes mettent en jeu la filtration et un traitement au chlore ou à

TABLEAU IV

Pourcentage de RADionucléides (présents dans l'eau brute de la Colombia River) QU'ON TROUVE APRÈS TRAITEMENT (floculation et filtration) [22] (en p. cent)

\begin{tabular}{|c|c|c|c|}
\hline${ }^{137} \mathrm{Cs}-{ }^{134} \mathrm{Cs} \ldots \ldots \ldots \ldots \ldots \ldots \ldots$ & 80 & ${ }^{22} \mathrm{Na}-{ }^{24} \mathrm{Na} \ldots \ldots \ldots \ldots \ldots \ldots \ldots$ & 90 \\
\hline${ }^{89} \mathrm{Sr}-{ }^{90} \mathrm{Sr} \ldots \ldots \ldots \ldots \ldots \ldots$ & 20 & ${ }^{s 1} \mathrm{Cr}, \ldots \ldots \ldots \ldots \ldots \ldots \ldots \ldots \ldots$ & 90 \\
\hline${ }^{103} \mathrm{Ru}{ }^{106} \mathrm{Ru} \ldots \ldots \ldots \ldots \ldots \ldots$ & 20 & ${ }^{54} \mathrm{Mn}, \ldots \ldots \ldots \ldots \ldots$ & 20 \\
\hline${ }^{131} \mathrm{I}-{ }^{129} \mathrm{I} \ldots \ldots \ldots \ldots \ldots$ & 80 & ${ }^{s s} \mathrm{Fe}-{ }^{s 9} \mathrm{Fe} \ldots \ldots \ldots \ldots \ldots \ldots \ldots \ldots$ & 20 \\
\hline 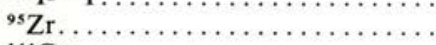 & 30 & 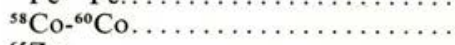 & 20 \\
\hline${ }^{144} \mathrm{Ce} \ldots \ldots \ldots \ldots \ldots \ldots \ldots$ & 20 & ${ }^{65} \mathrm{Zn} \ldots \ldots \ldots \ldots \ldots \ldots \ldots$ & 40 \\
\hline${ }^{3} \mathrm{H} \ldots \ldots \ldots \ldots \ldots \ldots$ & 100 & & \\
\hline
\end{tabular}


l'ozone (ou les deux successivement), ce qui peut conduire à des décontaminations (tableau IV) notamment pour les radionucléides à l'état colloïdal ou particulaire [22].

\section{Contamination du lait et des produits laitiers (vaches)}

Des études expérimentales entreprises en collaboration entre le C.E.A. et l'Institut National de la Recherche Agronomique (I.N.R.A.) ont montré que l'animal constitue un filtre très efficace vis-à-vis des polluants apportés par son alimentation (tableau V) $[13,17]$.

\section{TABLEAU $\mathrm{V}$}

\begin{tabular}{|l|l|}
\hline \multicolumn{2}{|c|}{ TAUX DE SECRÉTION LACTÉE DE DIVERS ÉLÉMENTS (en régime permanent) } \\
(élément sécrété en $1 . \mathrm{j}^{-1}$ élément ingéré en $1 . \mathrm{j}^{-1}$ )
\end{tabular}

\section{Contamination de la bière à partir de produits bruts contaminés}

A la suite d'études à l'échelon pilote, effectuées en collaboration entre le C.E.A. et le Centre de Brasserie-Malterie de la Faculté de Nancy, on a constaté que les isotopes des éléments présents dans les matières premières servant à la fabrication de la bière se retrouvent en presque totalité dans les drèches, la bière au stade de sa consommation étant très peu contaminée (tableau VI). Il y a cependant des exceptions relatives aux éléments présents dans le moût, en partie sous forme anionique ou électriquement neutres ( $\mathrm{Cr}$ en particulier) $[13,14]$.

\section{TABLEAU VI}

TENEURS EN QUELQUeS MÉTAUX DE LA BIÈRE (au stade de sa consommation) ET DES DRÊCHES DE BRASSERIE $\left(10^{-6}\right)$

\begin{tabular}{|c|c|c|}
\hline Éléments & Bière & Drêches \\
\hline 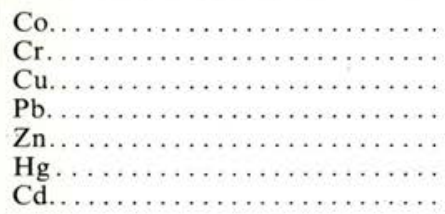 & $\begin{array}{l}0,02 \\
0,04 \\
0,04 \\
0,02 \\
0,4 \\
0,001 \\
0,006\end{array}$ & $\begin{array}{l}0,02 \\
0,01 \\
5,00 \\
0,1 \\
29 \\
0,80 \\
0,2\end{array}$ \\
\hline
\end{tabular}




\subsection{VAlORISATION DES DÉCHETS}

L'homme et les animaux domestiques éliminent un grand nombre de produits, déchets du métabolisme normal et fractions des produits bruts jugés impropres à la consommation directe. A une époque où on manque à la fois d'énergie utilisable et de protéines alimentaires, il est évidemment intéressant de réutiliser les divers déchets à ces différentes fins. Il est donc légitime de chercher à prévoir les conséquences possibles de telles valorisations des déchets. Il faut d'abord constater que, dans une société dite de consommation, l'homme écarte spontanément de son régime alimentaire précisément ce qui est le plus pollué, qu'il s'agisse de pollutions radioactives ou d'autres pollutions. La confrontation des teneurs en métaux lourds des drèches de brasseries et de la bière est significative (tableau VI)). La réutilisation des déchets recycle donc divers éléments ou composés indésirables sous une forme physicochimique où ils sont particulièrement mobiles.

La recherche de nouvelles sources de protéines pour l'homme pose des problèmes inhabituels, puisque, bien souvent, il s'agit là de " court-circuiter " les cheminements naturels des éléments et de leurs radionucléides par des transferts directs des déchets à l'homme sans passage par le milieu physique [5, $16,43]$. Très souvent les déchets servent de substrats à divers micro-organismes : algues, levures, bactéries qui, en se développant, constituent une source intéressante d'azote. En ce qui concerne les déchets d'origine végétale, une partie des radionucléides se trouvent simplement déposée à la surface des organes végétaux rejetés (feuilles, tiges, ...). Le développement des micro-organismes a pour résultat de métaboliser ces radionucléides, c'est-à-dire de les faire passer de l'état minéral à l'état organique, ce qui peut avoir une très grande incidence sur leur comportement ultérieur.

L'utilisation, pour l'alimentation humaine, de concentrés protéiniques d'origine animale, notamment de concentrés de protéines de poissons, est susceptible de poser de nouveaux problèmes, aussi bien dans le cadre des polluants non radioactifs que dans celui des polluants radioactifs [7]. Par exemple, le zinc 65 , polluant radioactif et le cadmium, polluant non radioactif, sont associés, chez les animaux aquatiques notamment, à des protéines et à des acides aminés soufrés de grand intérêt alimentaire.

\section{MICROLOCALISATION DES POLLUANTS}

Il est bien connu qu'il existe au sein des cellules des entités bien différenciées, parfois même bien délimitées et dont on a pu souvent trouver la fonction dans le métabolisme cellulaire. Il est, d'autre part, établi que les radionucléides, notamment ceux dont les comportements biochimiques sont voisins de ceux d'oligoéléments, se lient à des molécules de grand intérêt biologique : c'est par exemple, le cas des plutoniums qui présentent une grande affinité pour les ferritines qui représentent la forme protéique cellulaire (lysosomes) de stockage du fer [41]. Quoi qu'il en soit, pour les radionucléides émettant des rayonnements 
de TLE élevé et dont le parcours moyen est de l'ordre de la dimension des organelles subcellulaires ou des molécules, il peut en résulter une hétérogénéité très grande dans la dose délivrée localement. Une très récente étude de l'École de POLIKARPOV [31] concernant la microlocalisation intracellulaire de produits de

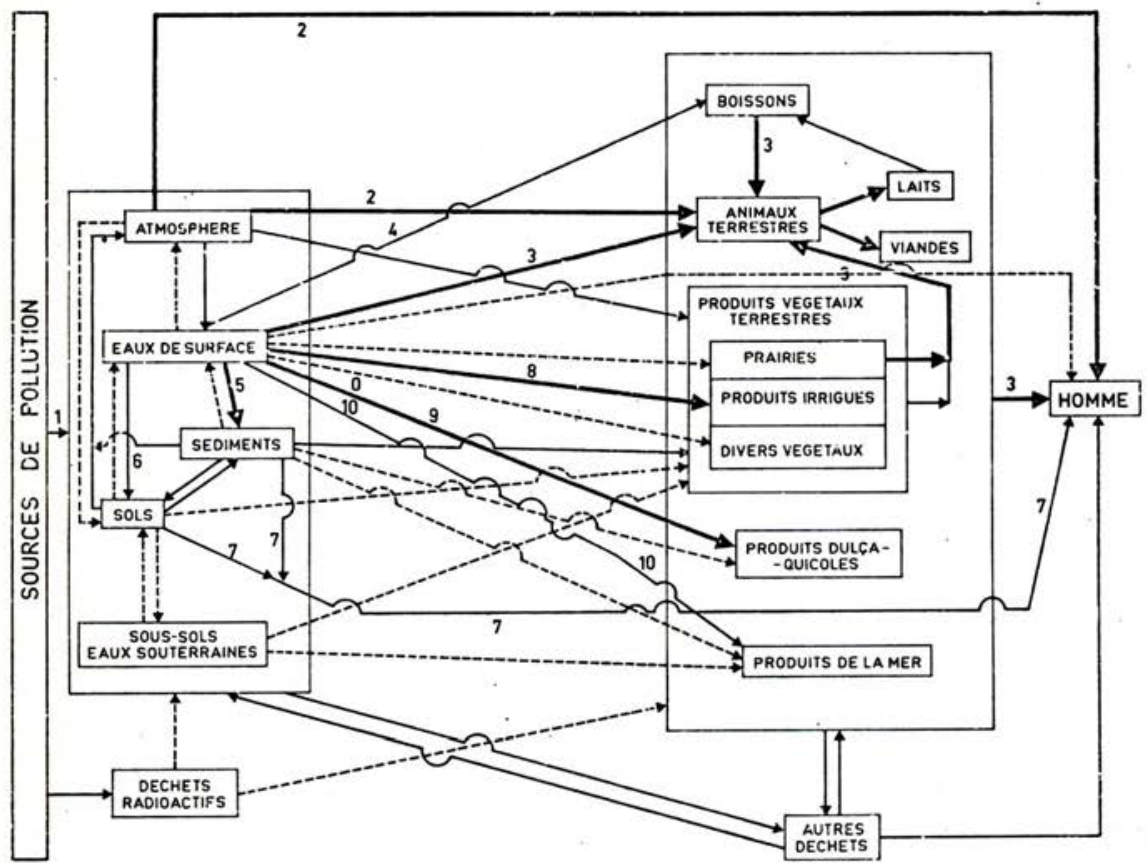

FIG. 1. - Transfert des radiocésiums.

Traits gras, transferts potentiels importants; traits pleins, transferts potentiels moyens; traits discontinus, transferts potentiels peu importants. 1, dispersion dans le milieu physique; 2 , inhalation directe par l'homme et les animaux terrestres supérieurs : fraction incorporée du Cs inhalé : $f_{a}=0,75 ; 3$, absorption intestinale par l'homme et les animaux terrestres supérieurs : fraction incorporée du Cs ingéré : $f_{e}: 0,5-0,8 ; 4$, transfert eaux brutes $\rightarrow$ eaux de boisson : $F \approx 0,8(0,1$ à 1$) ; 5$, transfert eaux de surface $\rightarrow$ sédiments : $K_{d}: 100$ à $500001 / \mathrm{kg}$, médiane eaux continentales : $10000 ; 6$, transfert eaux $\rightarrow$ sols : $F=5$ à 200 , médiane : $50 ; 7$, transfert direct par voie percutanée et par les blessures; 8 , transfert eaux d'irrigation $\rightarrow$ végétaux irrigués : pour les végétaux verts (aspersion) : $F=0,1$ à 0,7 , pour le riz (endosperme du grain) irrigué par submersion $F: 5$ à 40; 9 , transfert eaux continentales $\rightarrow$ poissons : $F: 100$ à 22000 , médiane : $3000 ; 10$, transfert eaux marines $\rightarrow$ produits de la mer : $F \approx 30 ; 3+11$, transfert aliment du bétail $\rightarrow$ lait : pour les vaches :

$$
d_{0}=\frac{\mathrm{Cs} / 1 \text { de lait }}{\text { Cs ingéré } / \text { jour }} \times 100 \approx 0,45 \text { à } 1,5
$$

$3+12$, transfert aliment du bétail $\rightarrow$ viande : pour les bovins :

$$
d_{1}=\frac{\mathrm{Cs} / \mathrm{kg} \text { de viande }}{\mathrm{Cs} \text { ingéré } / \text { jour }} \approx 2 \text {. }
$$


fission dans les cellules d'organismes dulçaquicoles, les characées, va dans le même sens. Des approches sont actuellement proposées en vue d'orienter les chercheurs vers des recherches microdosimétriques débouchant sur des conceptions nouvelles de la radioécologie appliquées aux animaux supérieurs, donc à l'homme, et au milieu naturel, le concept classique d'organe critique étant complété par celui d'organelle subcellulaire critique ou de molécule biochimique critique [15].

\section{CONCLUSION}

Cette étude, dont l'objet était de faire, le plus concrètement possible, le point des recherches sur les transferts des radionucléides, met l'accent sur un certain nombre d'aspects qui paraissent particulièrement importants actuellement, compte tenu de l'avancement des connaissances et de l'évolution prévisible de l'énergie nucléaire. Les problèmes soulevés ne sont certainement pas les seuls intéressants, mais il importait de se limiter. En particulier, l'incidence des irradiations externes et internes des organismes du milieu n'a pas été abordée ici, bien que son intérêt ne soit pas, bien entendu, mis en doute. Dans le cadre des effets de la radioactivité naturelle tellurique, des travaux comme ceux de DeLPouX, témoignent de ce fait [21].

Quoi qu'il en soit, et en se limitant aux problèmes de transfert, force est de constater que la radioécologie a fortement évolué, comme cela était naturel et souhaitable. Dans une première phase et pour mieux élaguer cette vaste discipline de problèmes alors difficilement solubles, on a cherché, peut-être trop rapidement, une quantification des transferts en faisant appel aux notions de facteurs de transfert et de dilution isotopique. On a ainsi débouché sur une structure de base scientifique et technique solide, qui a permis d'autres recherches, notamment sur la variabilité du facteur de transfert. L'avancement des disciplines nucléaires rend souhaitable une nouvelle progression, l'étude des transferts devenant difficilement dissociable de diverses disciplines fondamentales, en particulier de la biochimie moléculaire, de la toxicologie et de la microdosimétrie. Cependant, l'ensemble de ces recherches doit garder un objectif concret et être utilisable par les techniciens de la radioprotection lorsqu'ils ont à décider d'un site pour une nouvelle implantation. Les deux aspects, théorique et appliqué, doivent se compléter pour tendre à fournir aux utilisateurs des données simples, mais aussi complètes que possible ( fig. 1).

\section{BIBLIOGRAPHIE}

[1] Ancellin J., Avargues M., Bovard P., Guegueniat P., Vilquin A. Aspects biologiques et physicochimiques de la contamination radioactive d'espèces et de sédiments marins. Dans: Radioactive contamination of the marine environment, Seattle, 10-14 July 1972, Vienne, A.I.E.A., 1973, 225-243.

[2] Anspaugh L. R., Shinn J. H., Wilson D. W. Evaluation of the resuspension pathway toward protective guideline for soil contamination with radioactivity. Dans : Population 
dose evaluation and standards for man and his environment, Portoroz, 20-24 May 1974. Vienne. A.I.E.A., 1974, 513-524.

[3] Anspaugh L. R., Shinn J. H., Phelps P. L., Kennedy N. C. Resuspension and redistribution of plutonium in soils. Health Phys., 1975, 29, 571-582.

[4] AU F.H.F. The role of soil microorganisms in the movement of plutonium. Rapport américain NVO-AEIC-71-1, 1974.

[5] Autret. Les protéines non conventionnelles. C. R. Acad. Agr. France, 1972, 59, (8), 692-718.

[6] BARBER D. A. Effects on microorganisms on the absorption of inorganic nutrients by plants, Pesticide Sci., 1973, 4, 367-373.

[7] Beasley T. M., Jokela J. A., Eagle R. J. Radionuclides and selected trace elements in marine protein concentrates. Health Phys., 1971, 21, 815-820.

[8] BernhaRd M. Evaluation of the risks related to the discharge of radioactive isotopes in a marine environment. Rev. intern. Oceanogr. med., 1970, 20, 125-129.

[9] Bittel R. Étude de la solubilisation des éléments utiles aux végétaux en vue de l'appréciation de la fertilité minérale des sols. Thèse Doctorat ès Sciences Physiques, Paris, 1956.

[10] Bittel R. Incidence des paramètres physicochimiques sur la contamination radioactive des écosystèmes irrigués. Dans : Environmental behaviour of radionuclides released in the nuclear industry, Aix-en-Provence, 14-18 March 1973, Vienne, A.I.E.A., 1973, 291-302.

[11] Bittel R. A comparative study of two methods of estimating human internal exposure hazards resulting from the ingestion of sea foodstuffs. Dans : Health physics problems of internal contamination, IRPA 2nd European congress of radiation protection, Budapest, May 3-5, 1972 (BUJDOSO E., Ed.), Budapest, Akadémiai Kiado, 1973, 413-416.

[12] Bittel R. Les pollutions associées aux pollutions radioactives dans les écosystèmes aquatiques et irrigués. Radioprotection, 1974, 9, (2), 111-130.

[13] Bittel. R. Utilisation des études de radioprotection pour des recherches sur les transferts d'oligoéléments, du milieu aux produits végétaux et animaux. Dans : Nuclear techniques in animal production and health. Vienna, 2-6 Feb. 1976, Vienne, A.I.E.A., 1976, 17-23.

[14] Bittel R., Magnaval R. Étude du mouvement de polluants métalliques dans le milieu physique et les chaînes biologiques. Dans : Measurement, detection and control of environmental pollutants, Vienna, 15-19 March 1976, Vienne, A.I.E.A., 1976, 449-463.

[15] Bittel R., Magnaval R. Microlocalization of artificial radionuclides in radiological protection environment. Intern. Conference on molecular and microdistribution of radioisotopes and biological consequences, Jülich, Oct. 2-4, 1975, Curr. Top. Radiat. Res., 1977, 12.

[16] Bretagne Occidentale (Université de). Agriculture et alimentation. Contribution de l'Université de Bretagne Occidentale aux recherches concernant l'agriculture et l'alimentation (1970-1975) Brest, Université de Bretagne Occidentale et Quimper I.U.T. (Biologie), 1976.

[17] Bruant C., Bruant J. P., Neuburger M., Fourcy A., Vassal L., Disant C., Bittel R. Étude de quelques métaux lourds dans la chaîne herbe-produits laitiers au moyen de l'activation neutronique et de l'absorption atomique. Dans : Comparative studies of food and environmental contamination, Otaniemi, 27-31 Aug. 1973, Vienne, A.I.E.A., 1973, 293-307.

[18] Centre Technique de l'Élevage Bovin (ITEB-ITOVIC-ITP). L'élevage bovin et ses productions, statistiques, commentaires et documents pour la France et l'Europe des neufs. Paris, Réalisation SEGESA, 1973.

[19] Commission Internationale de Protection Radiologique. Recommandations. Publication CIPR 9. 
[20] Commission Internationale de Protection Radiologique. Les implications des recommandations de la Commission de maintenir les doses aux valeurs les plus faibles qu'il soit possible d'atteindre sans difficulté. Publication CIPR 22 (A. Duchene, trad.), 1974.

[21] Delpoux M. Étude expérimentale des effets de la radioactivité tellurique sur les végétaux. Thèse Doctorat ès Sciences Naturelles, Toulouse, 1974.

[22] Fletcher J. F., Dobson W. F. Hermes, a digital computer for estimating regional radioecological effects from the nuclear power industry. Rapport américain HEDL-TMF 71-168, 1971.

[23] Fraizier A., Ancellin J. Étude de la contamination de constituants du milieu marin par des formes solubles et insolubles de radionuclides. Dans : Impacts of nuclear releases into the aquatic environment, Otaniemi, 30 June-4 July 1975, Vienne, A.I.E.A., 1975, 49-62.

[24] Fraizier A., Ancellin J. Influence de la température sur la contamination d'espèces marines par le fer 59. Dans : Combined effects of radioactive, chemical and thermal releases to the environment. Stockholm, 2-5 June 1975, Vienne, A.I.E.A., 1975, 51-66.

[25] Fuchs H., Hoffmann W., von Euw H. M. Synergistic effects of airborne emissions from nuclear power plants. Dans : Combined effects of radioactive, chemical and thermal releases to the environment, Stockholm, 2-5 June 1975, Vienne, A.I.E.A., 1975, 243-252.

[26] Garnier A. Méthodologie d'évaluation des doses à la population résultant de rejets radioactifs dans l'atmosphère. Rapport CEA-R-4781, 1976.

[27] Guckert A., Breish H., Risinger O. Interface sol-racines. Étude au microscope électronique des relations mucigel-argile-micro-organismes. Soil Biol. Biochem., 1975, 7 (4/5). 241-251.

[28] Guegueniat P. Comportement physicochimique du ruthénium de fission dans le milieu marin. Thèse Doctorat ès Sciences Physiques. U.E.R. Sciences de la Matière, Université Louis-Pasteur, Strasbourg, 1974 et Rapport CEA-R.

[29] Hubschmann W. G., Nester K., Wilhelm J. G. Discussion of possible effects due to mixing of radioactive and thermal releases to the atmosphere from nuclear power plants. Dans : Combined effects of radioactive, chemical and thermal releases to the environment, Stockholm, 2-5 June 1975, Vienne, A.I.E.A., 1975, 233-242.

[30] KAYE S. V., Nelson D. J., Analysis of specific activity concept as related to environmental concentration of radionuclides. Nuclear Safety, 1968, 9, (1), 53-58.

[31] Marchyulenene D. P., Moteyunene E. B., Gudavichene N. A., Polikarpov G. G. Étude de l'accumulation sélective du césium 137, du strontium 90 et du césium 144 dans les compartiments cellulaires des Characées. Radioprotection, 1976, 11, 263-270.

[32] Metivier H. Contribution à l'étude de l'hydrolyse du plutonium tétravalent et de sa complexation par des acides d'intérêt biologique. Thèse Doctorat ès Sciences, Paris-VI, 1973, Rapport CEA-R-4477.

[33] Metivier H. Évolution physicochimique du mélange d'aérosols d'oxydes de sodium et de plutonium. Radioprotection, 1974, 9, (3), 187-191.

[34] Metivier H. Devenir biologique des mélanges d'aérosols d'oxyde de sodium et de plutonium. Radioprotection, 1976, 11, (4), 271-278.

[35] Mouillet L., Luquet F. M., Casalis J. Contribution à l'étude des variations de la teneur en sels minéraux du lait de vaches de diverses régions françaises. Le lait, 1975, 55, 683-694.

[36] Myttenaere C., Merlin M., Bittel R., Dabin P., Mousny J. M., Pozzi G. Influence du cadmium stable sur le transfert du zinc 65 en écosystème irrigué par submersion (rizière irriguée). Dans : Impacts of nuclear releases into the aquatic environment, Otaniemi, 30 July-4 July 1975, Vienne, A.I.E.A., 1975, 39-47.

[37] Pally M., Foulquier L. Introduction à l'étude de la fixation du cadmium par Anguilla (L.). Note CEA-N-1804, 1975. 
[38] Patel B., Balani M. C., Shakunt Patel, Panday V. K., Soman S. D. Impact of thermal and radioactive effluents on a tropical nearshore ecosystem. Dans : Combined effects of radioactive, chemical and thermal releases to the environment, Stockholm, 2-5 June 1975, Vienne, A.I.E.A., 1975, 17-34.

[39] Routson R. C., Wildung R. E., Garland T. R. The potential for plutonium complexation in soil and uptake by plants. U.S.A.E.C. report BNWL-2000, Pt 2, 1974, 21-36.

[40] Swanson J. L., Garland T. R., Wildung R. E. The potential for plutonium complexation in soils and uptake by plants. U.S.A.E.C. report BNWL-1950, Pt 2, 1974, 21-36.

[41] TAYLOR D. M. Interactions between transuranium elements and the components of cells and tissues. Health Phys., 1972, 22, 575-581.

[42] VAN WeERS A. W. The effects of temperature on the uptake and retention of ${ }^{60} \mathrm{Co}$ and ${ }^{65} \mathrm{Zn}$ by the common shrimp Crangon Crangon (L.). Dans : Combined effects of radioactive, chemical and thermal releases to the environment, Stockholm, 2-5 June 1975, Vienne, A.I.E.A., 1975, 35-42.

[43] Waslien C., Oswald W. Unusual sources of proteins for man. Crit. Rev. Food Sci. Nutr. (Cleveland), 1975, 6, (1), 77-151.

[44[ Waterloopkundig Laboratorium, Delft hydraulics Laboratory (in cooperation with Massachussetts Institute of Technology). European course on heat disposal from power generation in the water environment, Delft, Waterloopkundig laboratorium, 1975, 2 vol. 